\title{
New meniscal tears after $A C L$ injury: what is the risk? A systematic review protocol
}

\author{
Guri Ranum Ekås, ${ }^{1,2,3}$ Clare Ardern, ${ }^{4,5}$ Hege Grindem, ${ }^{6}$ Lars Engebretsen ${ }^{1,2,3}$
}

- Additional material is published online only. To view please visit the journal online (http://dx.doi.org/10.1136/ bjsports-2017-097728).

${ }^{1}$ Division of Orthopaedic Surgery, Oslo University Hospital, Oslo, Norway ${ }^{2}$ Oslo Sports Trauma Research Centre (OSTRC), Norwegian School of Sport Sciences, Oslo, Norway

${ }^{3}$ Institute of Clinical Medicine, University of Oslo, Oslo, Norway ${ }^{4}$ Division of Physiotherapy, Linkoping University, Linkoping, Sweden

${ }^{5}$ School of Allied Health, La Trobe University, Melbourne, Australia

${ }^{6}$ Department of Sports Medicine, Norwegian School of Sport Sciences, Oslo, Norway

\section{Correspondence to} Dr Guri Ranum Ekås, Oslo Sports Trauma Research Centre, Norwegian School of Sports Sciences, Pb 4014, Ullevål stadion, 0806 Oslo, Norway; g.r.ekas@nih.no

Revised 6 May 2017 Accepted 28 May 2017 Published Online First 24 June 2017

\section{ABSTRACT}

Background Secondary meniscal tears after $A C L$ injuries increase the risk of knee osteoarthritis. The current literature on secondary meniscal injuries after $A C L$ injury is not consistent and may have methodological shortcomings. This protocol describes the methods of a systematic review investigating the rate of secondary meniscal injuries in children and adults after treatment (operative or non-operative) for $A C L$ injury.

Methods We will search electronic databases (Embase, Ovid Medline, Cochrane, CINAHL (Cumulative Index to Nursing and Allied Health Literature), SPORTDiscus, PEDro and Google Scholar) from database inception. Extracted data will include demographic data, methodology, intervention details and patient outcomes. Risk of bias will be assessed using the Newcastle Ottawa checklist for cohort studies. Article screening, eligibility assessment, risk of bias assessment and data extraction will be performed in duplicate by independent reviewers. A proportion meta-analysis will be performed if studies are homogeneous $\left(I^{2}<75 \%\right)$. If meta-analysis is precluded, data will be synthesised descriptively using best-evidence synthesis. The strength of recommendations and quality of evidence will be assessed using the Grading of Recommendations Assessment Development and Evaluation working group methodology.

Ethics and dissemination This protocol is written according to the Preferred Reporting Items for Systematic reviews and Meta-Analyses, and was registered in the International Prospective Register of Systematic Reviews on 22 March 2016.

Trial registration number CRD42016036788.

\section{INTRODUCTION}

Preventing further injury to articular cartilage and menisci is a common justification to perform ACL reconstruction. ${ }^{1}$ Having an ACL reconstruction does not prevent knee osteoarthritis, ${ }^{2}{ }^{3}$ but meniscal injuries occurring either at the time of the index ACL injury or subsequent to the ACL injury increase the risk. ${ }^{4-6}$ Prevention of secondary meniscal injuries is highly relevant for all patients with ACL injury and their treating clinicians.

The ACL is a key stabilising structure in the knee, restricting tibial translation and rotation. ${ }^{78}$ The rationale for performing $\mathrm{ACL}$ reconstruction is that surgery stabilises the injured knee, preventing secondary meniscal injuries that may occur if the knee was to give way during physical activity or activities of daily living. In some countries, ACL reconstruction is a first-line treatment for almost all patients with ACL injury. ${ }^{9}{ }^{10}$ In other countries, including Norway ${ }^{11}{ }^{12}$ and Sweden, ${ }^{13}$ non-operative treatment is a more common approach to treating ACL injuries, even in physically active patients. In Sweden, between 2001 and 2009, 64\% of patients with ACL injury had non-operative treatment. ${ }^{13}$ In Norway, the absolute number of non-operated ACL injuries is unknown. However, it is estimated that approximately $50 \%$ of patients with ACL injury are treated non-operatively. ${ }^{14} 15$ Treatment choice (ACL reconstruction or non-operative treatment) is tailored to the individual, taking into consideration associated injuries and knee stability, as well as the patient's activity level and response to rehabilitation. The typical Scandinavian approach is that patients without additional injuries warranting surgery, who do not take part in pivoting sports and cope well after a trial of rehabilitation, are treated non-operatively with active rehabilitation. ${ }^{1112}$

In skeletally immature patients, Scandinavian clinicians are even more inclined to follow a non-operative approach than in adults. ${ }^{16}$ The rationale for non-operative treatment of ACL injuries in children is due to increased risk of growth disturbances related to the open growth plates ${ }^{17-19}$ and the thin, underdeveloped paediatric autograft that will not increase in size to match the growing individual. ${ }^{20}$ The graft may be at risk of reinjury over time. ${ }^{21}$ However, this approach is fiercely debated. ${ }^{22-24}$

Several cross-sectional studies report that delayed operative treatment for ACL injury is associated with higher rates of meniscal injuries. ${ }^{24-28}$ Conversely, prospective longitudinal studies show good results in both children and adults treated non-operatively. ${ }^{11} 1229$ A narrative review recently suggested higher rates of meniscal injury and early osteoarthritis in non-operatively treated children, ${ }^{30}$ while a systematic review of data from adults concluded that non-operative treatment with optional delayed surgery reduces the risk of knee osteoarthritis. ${ }^{31}$ Given the established link between secondary meniscal tears and knee osteoarthritis, ${ }^{4-6}$ the current literature does not seem to be consistent. Rates of meniscal injuries may differ across age and treatment groups, but inconsistencies could also arise if too much credence is given to study designs with a high risk of bias. ${ }^{22}$

We plan to investigate the existing evidence regarding secondary meniscal injury rates after ACL injury in both children and adults, and in patients who undergo different treatments (ACL reconstruction or active rehabilitation). We will also assess the risk of bias in relevant studies using a systematic approach. Conducting this systematic review is important to strengthen the knowledge background for clinical decision-making regarding treatment for ACL injury, and to help identify future research directions. 


\section{METHODS AND ANALYSIS}

This is a protocol of a systematic review adhering to the Preferred Reporting Items for Systematic reviews and Meta-Analysis (PRISMA) guidelines. ${ }^{32} 33$ The main aim of this systematic review is to investigate the rate of secondary meniscal injuries after ACL injury and to assess the quality of this body of evidence. The secondary aim is to describe the rates of secondary meniscal injury by treatment (non-operative or operative) and skeletal maturity (immaturity or maturity).

The following are the review questions:

1. What is the incidence of secondary meniscal injury following treatment for ACL injury?

2. Is there a difference in the incidence of secondary meniscal injury between patients who had ACL reconstruction and patients who had non-operative treatment?

3. Is there a difference in the incidence of secondary meniscal injury between patients who were skeletally immature and patients who were skeletally mature at the time of treatment for ACL injury?

A secondary meniscal injury is defined as a new meniscal injury that occurs after the ACL injury. To be able to differentiate between secondary meniscal injuries and meniscal injuries occurring concurrently with the ACL injury, studies must report the meniscal status at the time of diagnosis or at the start of treatment for ACL injury.

\section{Eligibility criteria}

We will apply the following inclusion criteria:

1. article includes patients with ACL injury (diagnosis must be confirmed with MRI and/or diagnostic arthroscopy), all age groups

2. article reports the number of meniscal injuries at the time of ACL injury diagnosis or at start of index treatment for ACL injury to establish baseline meniscal status

3. article reports the number of secondary (new) meniscal injuries that have occurred since the defined baseline meniscal status

4. original research article

5. article includes a minimum of 20 participants at final follow-up

6. published in English language.

We will apply the following exclusion criteria:

1. systematic reviews, meta-analyses or other types of literature review

2. only reporting meniscal injury at the time of ACL injury diagnosis or at the start of treatment

3. case studies

4. non-human studies

5. specifying the index treatment as revision ACL surgery

6. only including patients with knee dislocation or multiligament knee injuries (rupture of more than 2 of 4 main stabilising structures).

Studies exclusively evaluating patients undergoing revision ACL surgery or patients with knee dislocations or multiligament injuries will be excluded because they include selected groups of patients with worse outcomes and higher failure rates. ${ }^{34}$

\section{Search strategy}

In collaboration with two university librarians, we developed search strategies for the Embase, Ovid Medline, Cochrane Library, CINAHL, SPORTDiscus and PEDro electronic databases, adapting the search strategies to medical subject headings terms and keywords as necessary for each database (see online supplementary file 1 for the search strategy as applied to the Ovid Medline database). A pilot of the systematic search was conducted on 2 February 2016 by a librarian. There are no limits to language, publication year or study design applied to the search. An additional search will be conducted in Google Scholar. We will rerun the searches prior to manuscript submission to identify any eligible studies published since our first database search.

To supplement the electronic database search, we will handsearch the reference lists of included studies and relevant systematic reviews for potentially eligible articles missed in the electronic database search, and conduct forward citation tracking using Google Scholar. We will also hand-search the ePublication lists for any newly published articles that may not be indexed by the electronic databases in the following journals: American Journal of Sports Medicine, Journal of Bone and Joint Surgery, Arthroscopy, Bone \& Joint Journal and the British Journal of Sports Medicine.

All search results will be exported to an EndNote library, where we will check for, and exclude, duplicate records. All included articles will be uploaded to Covidence for article screening (covidence.org). Covidence is an internet-based software program specifically designed for managing article selection and data extraction in systematic reviews.

\section{Article selection}

Two reviewers (GRE and CA) will independently screen titles and abstracts to identify potentially relevant studies. The fulltext version of all articles identified as potentially relevant will be retrieved for eligibility assessment. If it is unclear from the title and abstract screen whether an article meets the inclusion criteria, the article will be reviewed in full text.

Two independent reviewers (GRE and CA) will screen the fulltext articles to evaluate whether the studies meet the selection criteria. Any disagreements will be resolved by consensus discussion. If an agreement regarding eligibility cannot be reached, a third author (HG) will be consulted. The inter-rater agreement (kappa) for eligibility assessment will be calculated and reported. The reasons for excluding studies will be documented.

\section{Data extraction}

Data from all included articles will be extracted independently and in duplicate using a data extraction template specifically created for this review (GRE will extract data from all included articles; CA and HG will extract data from $50 \%$ of the articles each). Disagreements will be resolved via consensus discussion. A third reviewer (either CA or HG) will be consulted if discrepancy cannot be resolved. If required, we will contact study authors by email for clarification. The data extraction form will be piloted and tested by the reviewers (GRE, HG and CA) prior to the data extraction process to ensure consistency. The predefined variables for extraction are the following:

1. publication details: first author, year, funding source

2. population: age, skeletal maturity, method for determining skeletal maturity, sex, activity level (before and after injury), population setting (where the patients are recruited from)

3. intervention: ACL reconstruction (surgical technique, graft source, timing), non-operative treatment (rehabilitation protocol), recommendation of activity modification

4. outcome: number of meniscal injuries at baseline (medial, lateral or both), number of meniscal injuries at follow-up (medial, lateral or both), how secondary injuries were assessed and diagnosed (eg, patient-reported symptoms, follow-up 
arthroscopy), diagnostic method (eg, MRI or arthroscopy), return to sport, giving way episodes after baseline

5. methodology: study design, sample size, length of follow-up.

\section{Risk of bias assessment}

Because we have not limited our review to a particular study design, the risk of bias assessment must be appropriate for randomised and non-randomised studies. Non-comparative studies are also pertinent to answering our primary review question. As summarised in the PRISMA elaboration, a scale that numerically summarises risk of bias assessment into a single score on a continuous scale can be misleading, or at least, unhelpful. ${ }^{35}$ We pilot-tested the Cochrane Tool to Assess Risk of Bias in Cohort Studies ${ }^{36}$ and the Newcastle Ottawa Quality Assessment Scale for Cohort Studies. ${ }^{37}$ Of the two, we found the Newcastle Ottawa Scale better suited our primary review question and the expected variation in study design.

The risk of bias assessment will be used to inform our data synthesis and the conclusions that we draw.

Two independent reviewers will perform the risk of bias assessment using the Newcastle Ottawa Scale (GRE will assess all included articles; CA and HG will assess 50\% of the articles each). Disagreements will be resolved via consensus discussion. A third reviewer (either CA or HG) will be consulted if discrepancy cannot be resolved.

\section{Data analysis and presentation}

If there are sufficient data, we will conduct a proportion meta-analysis to determine the rate of secondary meniscal injury following treatment for ACL injury. If studies are sufficiently homogeneous $\left(\mathrm{I}^{2}<75 \%\right)$, we will conduct a meta-analysis using a random-effects model. We will present summary estimates in forest plots. If the $\mathrm{I}^{2}$ is greater than $50 \%$, possible sources of heterogeneity will be explored via subgroup analyses. Predefined subgroups are patients treated with ACL reconstruction compared with non-operative treatment, and data from skeletally immature patients compared with skeletally mature patients. We will conduct a sensitivity analysis to assess whether differences in diagnostic methods affect the results. If there are sufficient data, we will conduct meta-regression to examine the relationship between age and secondary meniscal injury. If possible, we will look at the relationship between activity level, preinjury and post-treatment, and secondary meniscal injury. We will assess publication bias and small study effects using a funnel plot for outcomes where there are 10 or more comparisons. ${ }^{36}$ Stata 14.2 will be used for all statistical calculations.

Due to diversity in our study populations, different timing and techniques used for ACL reconstruction, inclusion of studies also evaluating non-operative treatment and different study designs, performing a meta-analysis may not be possible. If meta-analysis is precluded, we will conduct a descriptive synthesis using a best-evidence synthesis approach.

\section{Strength of recommendations and quality of evidence}

We will assess the strength of recommendations based on the Grading of Recommendations Assessment Development and Evaluation (GRADE) working group methodology (www.gradeworkinggroup.org). ${ }^{38}$ The two categories weak/conditional evidence and strong evidence will be used.

The quality of evidence for the study outcome, secondary meniscal injuries, will also be judged using the GRADE methodology. ${ }^{38}$ The quality of evidence will be assessed according to the domains of risk of bias, consistency, directness, precision and publication bias. Quality will be graded as high, moderate, low or very low as defined according to the GRADE working group. ${ }^{38-40}$

\section{Ethics and dissemination}

No primary data will be collected. The systematic review article will be submitted for publication in a peer-reviewed journal. The findings will also be included in a $\mathrm{PhD}$ thesis and disseminated at international sports medicine and orthopaedic conferences.

\section{Trial registration number}

The protocol is registered in the International Prospective Register of Systematic Reviews (CRD42016036788).

Key messages

- Preventing further injury to articular cartilage and menisci is a common justification to perform ACL reconstruction.

- The current literature regarding secondary (new) meniscal injury rates after $A C L$ injury does not seem to be consistent and may have methodological shortcomings.

- Rates of secondary meniscal tears after ACL injury may differ across age and treatment groups, but inconsistencies could also arise if too much credence is given to study designs with a high risk of bias.

Acknowledgements Librarians, Marte Ødegaard (at the University of Oslo Medical Library) and Elin Hecker (at the Norwegian School of Sports Sciences Library) helped to develop and conduct search strategies.

Contributors GRE is the guarantor and first author of the protocol. LE is her PhD supervisor and has provided advice throughout the process of planning this protocol. GRE, HG and CA drafted the manuscript and outlined eligibility criteria, risk of bias assessment strategy and data extraction criteria. LE reviewed and commented on the manuscript. All authors have contributed to the final design of the protocol. All authors have read this manuscript, provided feedback and taken part in the revision process. All authors have approved the final manuscript.

Funding Oslo Sports Trauma Research Center (OSTRC) supports the initiative of this systematic review. GRE is employed by OSTRC during her PhD project. OSTRC can therefore be regarded as the sponsor of this systematic review, but no additional funding has been received for this systematic review. OSTRC is an independent and non-profit research institution focusing on sports trauma and injury prevention research. OSTRC will have no input on the interpretation or publication of the study results.

Competing interests LE has received fellowship support and research grants from Arthrex and Smith \& Nephew. The authors have no further competing interests to disclose.

Provenance and peer review Not commissioned; externally peer reviewed. (c) Article author(s) (or their employer(s) unless otherwise stated in the text of the article) 2018. All rights reserved. No commercial use is permitted unless otherwise expressly granted.

\section{REFERENCES}

1 Richmond JC, Lubowitz JH, Poehling GG. Prompt operative intervention reduces long-term osteoarthritis after knee anterior cruciate ligament tear. Arthroscopy 2011:27:149-52.

2 Øiestad BE, Engebretsen L, Storheim K, et al. Knee osteoarthritis after anterior cruciate ligament injury: a systematic review. Am J Sports Med 2009;37:1434-43.

3 Risberg MA, Oiestad BE, Gunderson R, et al. Changes in Knee Osteoarthritis, Symptoms, and Function After Anterior Cruciate Ligament Reconstruction: A 20-Year Prospective Follow-up Study. Am J Sports Med 2016;44.

4 Oiestad BE, Holm I, Aune AK, et al. Knee function and prevalence of knee osteoarthritis after anterior cruciate ligament reconstruction: a prospective study with 10 to 15 years of follow-up. Am J Sports Med 2010;38:2201-10.

5 van Meer BL, Meuffels DE, van Eijsden WA, et al. Which determinants predict tibiofemoral and patellofemoral osteoarthritis after anterior cruciate ligament injury? A systematic review. Br J Sports Med 2015;49:975-83. 
6 van Meer BL, Oei EH, Meuffels DE, et al. Degenerative Changes in the Knee 2 Years After Anterior Cruciate Ligament Rupture and Related Risk Factors: A Prospective Observational Follow-up Study. Am J Sports Med 2016;44:1524-33.

7 Zlotnicki JP, Naendrup JH, Ferrer GA, et al. Basic biomechanic principles of knee instability. Curr Rev Musculoskelet Med 2016;9:114-22.

8 Ellison AE, Berg EE, Embryology BEE. Embryology, anatomy, and function of the anterior cruciate ligament. Orthop Clin North Am 1985;16:3-14.

9 Sanders TL, Maradit Kremers H, Bryan AJ, et al. Incidence of Anterior Cruciate Ligament Tears and Reconstruction: a 21-Year Population-Based Study. Am J Sports Med 2016;44:1502-7.

10 AAOS. Summary of recommendations: management of anterior cruciate ligament injuries. http://www.aaos.org/cc_files/aaosnow/oct14/clinical5_t1.pdf.

11 Grindem H, Eitzen I, Engebretsen L, et al. Nonsurgical or Surgical Treatment of ACL Injuries: Knee Function, Sports Participation, and Knee Reinjury: The Delaware-Oslo ACL Cohort Study. J Bone Joint Surg Am 2014;96:1233-41.

12 Moksnes H, Engebretsen L, Eitzen I, et al. Functional outcomes following a nonoperative treatment algorithm for anterior cruciate ligament injuries in skeletally immature children 12 years and younger. A prospective cohort with 2 years follow-up. Br J Sports Med 2013:47:488-94.

13 Nordenvall R, Bahmanyar S, Adami J, et al. A population-based nationwide study of cruciate ligament injury in Sweden, 2001-2009: incidence, treatment, and sex differences. Am J Sports Med 2012;40:1808-13.

14 Granan LP, Bahr R, Steindal K, et al. Development of a national cruciate ligament surgery registry: the Norwegian National Knee Ligament Registry. Am J Sports Med 2008;36:308-15.

15 Ekas GR, Engebretsen L. In process Citation]. Tidsskr Nor Laegeforen 2016:136:298.

16 Moksnes H, Engebretsen L, Risberg MA. Management of anterior cruciate ligament injuries in skeletally immature individuals. J Orthop Sports Phys Ther 2012;42:172-83.

17 Chotel F, Henry J, Seil R, et al. Growth disturbances without growth arrest after ACL reconstruction in children. Knee Surg Sports Traumatol Arthrosc 2010;18:1496-500.

18 Chotel F, Seil R. Growth disturbances after transphyseal ACL reconstruction in skeletally immature patients: who is more at risk? Young child or adolescent? J Pediatr Orthop 2013;33:585-6.

19 Moksnes H, Engebretsen L, Seil R. The ESSKA paediatric anterior cruciate ligament monitoring initiative. Knee Surg Sports Traumatol Arthrosc 2016;24.

20 Astur DC, Arliani GG, Debieux P, et al. Intraarticular hamstring graft diameter decreases with continuing knee growth after $\mathrm{ACL}$ reconstruction with open physes. Knee Surg Sports Traumatol Arthrosc 2016;24:792-5.

21 Magnussen RA, Lawrence JT, West RL, et al. Graft size and patient age are predictors of early revision after anterior cruciate ligament reconstruction with hamstring autograft. Arthroscopy 2012;28:526-31.

22 Moksnes $H$, Engebretsen L, Risberg MA. The current evidence for treatment of ACL injuries in children is low: a systematic review. J Bone Joint Surg Am 2012;94:1112-9.

23 Siebold R, Seil R, Engebretsen L. ACL tear in kids: serious injury with high risk of osteoarthritis. Knee Surg Sports Traumatol Arthrosc 2016;24:641-3.
24 Anderson AF, Anderson CN. Correlation of meniscal and articular cartilage injuries in children and adolescents with timing of anterior cruciate ligament reconstruction. $A m$ J Sports Med 2015;43:275-81.

25 Brambilla L, Pulici L, Carimati G, et al. Prevalence of Associated Lesions in Anterior Cruciate Ligament Reconstruction: Correlation With Surgical Timing and With Patient Age, Sex, and Body Mass Index. Am J Sports Med 2015;43:2966-73.

26 Granan LP, Bahr R, Lie SA, et al. Timing of anterior cruciate ligament reconstructive surgery and risk of cartilage lesions and meniscal tears: a cohort study based on the Norwegian National Knee Ligament Registry. Am J Sports Med 2009;37:955-61.

27 Lawrence JT, Argawal N, Ganley TJ. Degeneration of the knee joint in skeletally immature patients with a diagnosis of an anterior cruciate ligament tear: is there harm in delay of treatment? Am J Sports Med 2011;39:2582-7.

28 Ralles S, Agel J, Obermeier M, et al. Incidence of secondary Intra-articular injuries with Time to Anterior Cruciate Ligament reconstruction. Am J Sports Med 2015;43:1373-9.

29 Frobell RB, Roos HP, Roos EM, et al. Treatment for acute anterior cruciate ligament tear: five year outcome of randomised trial. Br J Sports Med 2015;49:700.

30 Anderson CN, Anderson AF. Management of the Anterior Cruciate Ligament-Injured knee in the Skeletally immature athlete. Clin Sports Med 2017;36:35-52.

31 Duncan KJ, Chopp-Hurley JN, Maly MR. A systematic review to evaluate exercise for anterior cruciate ligament injuries: does this approach reduce the incidence of knee osteoarthritis? Open Access Rheumatol 2016;8:1-16.

32 Moher $D$, Shamseer $L$, Clarke $M$, et al. Preferred reporting items for systematic review and meta-analysis protocols (PRISMA-P) 2015 statement. Syst Rev 2015;4:1.

33 Shamseer L, Moher D, Clarke M, et al. Preferred reporting items for systematic review and meta-analysis protocols (PRISMA-P) 2015: elaboration and explanation. BMJ 2015;349:g7647.

34 Wright RW, Gill CS, Chen L, et al. Outcome of revision anterior cruciate ligament reconstruction: a systematic review. J Bone Joint Surg Am 2012;94:531-6.

35 Jüni $P$, Witschi $A$, Bloch $R$, et al. The hazards of scoring the quality of clinical trials for meta-analysis. JAMA 1999;282:1054-60.

36 Cochrane. Higgins JPT, Green S, eds. Cochrane Handbook for Systematic Reviews of Interventions Version 5.1.0. 2011. www.handbook.cochrane.org. [updated March 2011].

37 Wells GA, Shea B, Higgins JP, et al. Checklists of methodological issues for review authors to consider when including non-randomized studies in systematic reviews. Res Synth Methods 2013;4:63-77.

38 GRADE 2006 http://www.gradeworkinggroup.org/.

39 Alonso-Coello P, Schünemann HJ, Moberg J, et al. GRADE Working Group. GRADE Evidence to Decision (EtD) frameworks: a systematic and transparent approach to making well informed healthcare choices. 1: Introduction. BMJ 2016;353:i2016.

40 Alonso-Coello P, Oxman AD, Moberg J, et al. GRADE evidence to decision (EtD) frameworks: a systematic and transparent approach to making well informed healthcare choices. 2: clinical practice guidelines. BMJ 2016;353:i2089. 